\title{
Simultaneous Analysis of Seven Benzodiazepines in Dietary Supplements as Adulterants Using High Performance Liquid Chromatography and its Application to an Identification System for Diazepam
}

\author{
Eiichi Mikami, ${ }^{*, a}$ Tomomi Goto, ${ }^{a}$ Tsutomu Ohno, ${ }^{a}$ Hisao Oka, ${ }^{a}$ and Hisayuki Kanamori ${ }^{b}$ \\ ${ }^{a}$ Aichi Prefectural Institute of Public Health, 7-6 Nagare, Tsuji-machi, Kita-ku, Nagoya 462-8576, Japan and ${ }^{b}$ Hiroshima Prefectural \\ Health Environment Center, 1-6-29 Minami-machi, Minami-ku, Hiroshima 734-0007, Japan
}

(Received August 24, 2004; Accepted January 19, 2005)

\begin{abstract}
A high performance liquid chromatography (HPLC) method for the simultaneous analysis of the seven benzodiazepines (BZDs), oxazolam, nitrazepam, oxazepam, tofisopam, triazolam, clotiazepam and diazepam (DIA) is presented for application in the screening examination of dietary supplements as adulterants. Samples were analyzed after extraction with methanol. HPLC analysis was performed with on a column of Wakosil 5 C18 $(4.6 \times$ $150 \mathrm{~mm}, 5 \mu \mathrm{m}$ ) with the mobile phase consisting of 1-heptanesulfonic acid sodium salt $5 \mathrm{mM}$ dissolved in water/ acetonitrile $1000 \mathrm{ml}(13: 7, \mathrm{v} / \mathrm{v})$, adjusted with phosphoric acid to $\mathrm{pH} 2.4$. The column eluent was monitored with a photodiode array detector, and the quantitative analysis of BZDs was performed at $225 \mathrm{~nm}$. The calibration curves of the seven BZDs showed good linearity and the correlation coefficients were better than 0.999 in all cases. When this procedure was applied to commercial supplements, an DIA-adulterated capsule-type supplement was detected. Finally, DIA was identified and determined using a combination of three different analytical methods: HPLC/photodiode array, thin-layer chromatography (TLC), and LC/MS. DIA was present at a concentration of $1.9 \mathrm{mg} / \mathrm{capsule.}$ The procedure described here can provide a broad analysis in a single run within 17 min and is available for the screening of seven BZDs in adulterated supplements with minimal sample preparation.
\end{abstract}

Key words — dietary supplement, benzodiazepine, diazepam, HPLC

\section{INTRODUCTION}

Dietary supplements have recently been gaining popularity with increasing health awareness among the Japanese. The presence of therapeutic medicinal ingredients intentionally added to supplements has been reported. ${ }^{1-5)}$ Such supplements are worrying and, without prior knowledge of the addition of drugs, potential hazards to health cannot easily be avoided. For surveillance programs, the establishment of a more effective screening procedure for medicinal ingredients in supplements is required..$^{6-8)}$

Benzodiazepines (BZDs) are most frequently prescribed as sedatives and hypnotics and patients

\footnotetext{
*To whom correspondence should be addressed: Aichi Prefectural Institute of Public Health, 7-6 Nagare, Tsuji-machi, Kitaku, Nagoya 462-8576, Japan. Tel.: +81-52-910-5629; Fax: +8152-913-3641; E-mail: eiichi_mikami@ pref.aichi.lg.jp
}

are easily subject to intoxication due to accidental overdose or misuse. A large number of analytical methods have been published for the determination of BZDs. Among these various chromatographic techniques, GC/MS, ${ }^{9,10)}$ HPLC, ${ }^{11,12)}$ and $\mathrm{LC} \mathrm{MS}^{13)}$ are commonly used for analyzing BZDs. However, they are not suitable to analyze BZDs in adulterated dietary supplements when considering single oral doses of marketed BZDs and minimizing sample preparation for periodic inspection. There is a need for the development of a new screening method that determines and identifies most common BZDs using simple, low-cost instrumentation.

The present paper describes an HPLC method for the simultaneous analysis of seven BZDs: oxazolam (OXO), nitrazepam (NIT), oxazepam (OXE), tofisopam (TOF), triazolam (TRI), clotiazepam (CLO), and diazepam (DIA) as adulterants in dietary supplements. Finally, an DIA-adulterated capsule-type supplement was identified and 
determined using a combination of three different analytical methods: HPLC/photodiode array, thinlayer chromatography (TLC), and LC/MS.

\section{MATERIALS AND METHODS}

Samples — Fourteen supplements (six of the tablet type, eight of the capsule type) were collected in Nagoya (Japan) markets in 2003. One capsuletype supplement was offered to an inhabitant of Hiroshima prefecture (Japan), who received it from a traveler to China.

Chemicals and Instrumentation - OXO, NIT, TOF, TRI, CLO, and DIA were of Japanese Pharmacopoeia (14th ed.) quality. OXE was of Japanese Pharmaceutical Codex (2002) quality. 1-Heptanesulfonic acid sodium salt (HSA) and HPLC-grade acetonitrile were purchased from Wako Pure Chemical Industries (Osaka, Japan). All other reagents and organic solvents were of analytical grade. HPLC/ photodiode array analyses were carried out using a Shimadzu CLASS-VP Series (Kyoto, Japan). LC/ MS with electrospray-ionization (ESI) interface analysis was carried out using a Micromass LCT mass spectrometer (Manchester, U.K.) to the effluent from an Agilent HP1100 LC system (Palo Alto, CA, U.S.A.).

Sample Preparation — A powdered sample of a single intake was transferred to a $100-\mathrm{ml}$ volumetric flask. The volume was adjusted with methanol, and the solution was sonicated for $60 \mathrm{~min}$ in an ultrasonic bath. Portions of these solutions were then filtered through a 0.5- $\mu \mathrm{m}$ PTFE filter (Toyo Roshi Kaisha, Tokyo, Japan). An aliquot (10 $\mu$ l) of each solution was injected onto the column.

HPLC Operating Conditions - HPLC analysis was performed with a column of Wakosil 5C18 ( $5 \mu \mathrm{m}, 4.6 \times 150 \mathrm{~mm}$, Wako Pure Chemical Industries), at a flow rate of $1.0 \mathrm{ml} / \mathrm{min}$. The mobile phase consisted of HSA $5 \mathrm{mM}$ dissolved in $1000 \mathrm{ml}$ of water/acetonitrile (13:7, v/v), adjusted with phosphoric acid to $\mathrm{pH} 2.4$. The column temperature was $40^{\circ} \mathrm{C}$ and the eluent was monitored using a photodiode array detector (220-340 $\mathrm{nm}$ ). The sample solution $(10 \mu \mathrm{l})$ was applied to quantitative analysis at $225 \mathrm{~nm}$ using a detector range of 0.08 aufs.

Calibration Curves of Standard Solutions Standard stock solutions of OXO $(0.2 \mathrm{mg} / \mathrm{ml})$, NIT $(0.05 \mathrm{mg} / \mathrm{ml})$, OXE $(0.05 \mathrm{mg} / \mathrm{ml})$, TOF $(0.12 \mathrm{mg} /$ $\mathrm{ml})$, TRI $(0.01 \mathrm{mg} / \mathrm{ml})$, CLO $(0.1 \mathrm{mg} / \mathrm{ml})$, and DIA $(0.04 \mathrm{mg} / \mathrm{ml})$ were prepared by dissolving appropri- ate amounts of the respective BZDs in methanol. BZD standard stock solutions $(0.5,1,2,3$, and $4 \mathrm{ml})$ were pipetted into $10-\mathrm{ml}$ volumetric flasks and made up with methanol to the final volume. BZD mixed standard solutions of OXO $(10-80 \mu \mathrm{g} / \mathrm{ml})$, NIT (2.5$20 \mu \mathrm{g} / \mathrm{ml})$, OXE (2.5-20 $\mu \mathrm{g} / \mathrm{ml})$, TOF $(6-50 \mu \mathrm{g} / \mathrm{ml})$, TRI $(0.5-4 \mu \mathrm{g} / \mathrm{ml}), \mathrm{CLO}(5-40 \mu \mathrm{g} / \mathrm{ml})$, and DIA (2$16 \mu \mathrm{g} / \mathrm{ml})$ were prepared. An aliquot $(10 \mu \mathrm{l})$ of each standard solution was injected. All measurements were performed in duplicate for each concentration. The peak areas were measured and those of the analytes (y) were plotted against the respective concentration $(\mu \mathrm{g} / \mathrm{ml})$ of BZDs $(\mathrm{x})$. Least-square linear regression analysis was used to determine the slope, $y$-intercept, and correlation coefficients of the standard plots.

\section{Identification System for DIA -}

$T L C$ : The sample solution $(20 \mu \mathrm{l})$ was applied to silica gel $60 \mathrm{~F}_{254}$ plates (Merck, Darmstadt, Germany) with hexane/ethyl acetate $(1: 1)$ as the developing solvent. ${ }^{14)}$ Spots were located under UV light at $254 \mathrm{~nm}$.

ESI-LC/MS: Mass spectra of DIA in LC/MS were investigated with the ESI interface under positive-ion mode. The operating parameters were: electrospray voltage, $3 \mathrm{kV}$; cone voltage, $30 \mathrm{~V}$; ion source temperature, $150^{\circ} \mathrm{C}$; and desolvation chamber temperature, $250^{\circ} \mathrm{C}$. LC operating conditions for the MS system were carried out on a column of Cosmosil 5C18-MS (5 $\mu \mathrm{m}, 4.6 \times 150 \mathrm{~mm}$, Nacalai Tesque, Kyoto, Japan) with water/acetonitrile ( $1: 1$, $\mathrm{v} / \mathrm{v})$ as the mobile phase, at a flow rate of $0.8 \mathrm{ml} / \mathrm{min}$.

\section{RESULTS AND DISCUSSION}

\section{Optimization of the Chromatographic System}

The seven BZDs were insufficiently separated on the reverse-phase column when the mobile phase consisted of acetonitrile/water. They showed reduced resolution and it hampered their accurate determination. The addition of HSA, the hydrophobic ionpair reagent, is particularly useful for the separation and detection of several drugs, such as nabumetone ${ }^{15)}$ and mosapride, ${ }^{16)}$ because it provides appropriate capacity factors for complexes formed with the counterion. When the effects of HSA concentration were examined, as shown in Fig. 1A, the capacity factor of the analyte was enhanced by increasing the HSA concentration from 1.25 to $7.5 \mathrm{mM}$. A concentration of $5 \mathrm{mM}$ was selected due to its optimum peak shape and resolution. The effects of mobile 

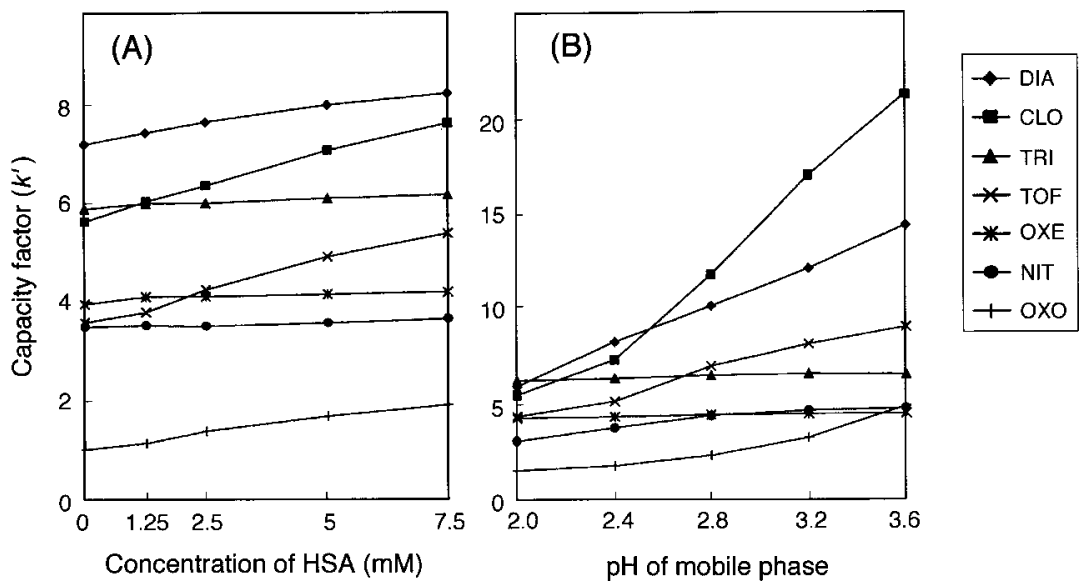

Fig. 1. Effects of (A) HSA Concentration and (B) $\mathrm{pH}$ on the Capacity Factor of Benzodiazepines

(A) The mobile phase was water/acetonitrile (13:7, v/v), adjusted with phosphoric acid to $\mathrm{pH}$ 2.4. (B) The mobile phase was HSA $5 \mathrm{mM}$ dissolved in water/acetonitrile $1000 \mathrm{ml}(13: 7, \mathrm{v} / \mathrm{v})$.

phase $\mathrm{pH}$ from 2.0 to 3.6 on the analyte retention were examined, in which seven BZDs were eluted within 20 min. As shown in Fig. 1B, CLO and DIA were strongly retained on the column. A pH value of 2.4 in the mobile phase was selected due to its optimum peak resolution and elution of the compounds within $17 \mathrm{~min}$. Utilizing chromatographic conditions, the capacity factors were 1.7 (OXO), 3.5 (NIT), 4.1(OXE), 5.1 (TOF), 6.1 (TRI), 7.2 (CLO), and 8.1 (DIA), respectively. When six other BZDs (ethyl loflazepate, flutazolam, haloxazolam, lormetazepam, midazolam, prazepam) were examined under these HPLC conditions, ethyl loflazepate, haloxazolam, lormetazepam, midazolam, and prazepam were eluted over $20 \mathrm{~min}$, and flutazolam displayed a broad peak.

An HPLC system equipped with a photodiode array detector was used because it provided a more definite identification than the retention index alone for effective screening. Figure 2 shows the UV spectra of the seven monitored BZD standards. The detector wavelength for BZDs in quantitative analysis was $225 \mathrm{~nm}$, which showed higher sensitivity.

\section{Analytical Performance Characteristics}

The calibration curves of BZDs in the concentration $(\mu \mathrm{g} / \mathrm{ml})$ ranges of 10-80 (OXO), 2.5-20 (NIT, OXE), 6-50 (TOF), 0.5-50 (TRI), 5-40 (CLO), and 2-16 (DIA) showed good linearity with a detection wavelength of $225 \mathrm{~nm}$ at a sensitivity of 0.08 aufs. The correlation coefficients were better than 0.999 in all cases. The intercepts with the y-axis were not markedly different from the origin. The instrumental detection limits $(\mu \mathrm{g} / \mathrm{ml})$, based on a signal-to-

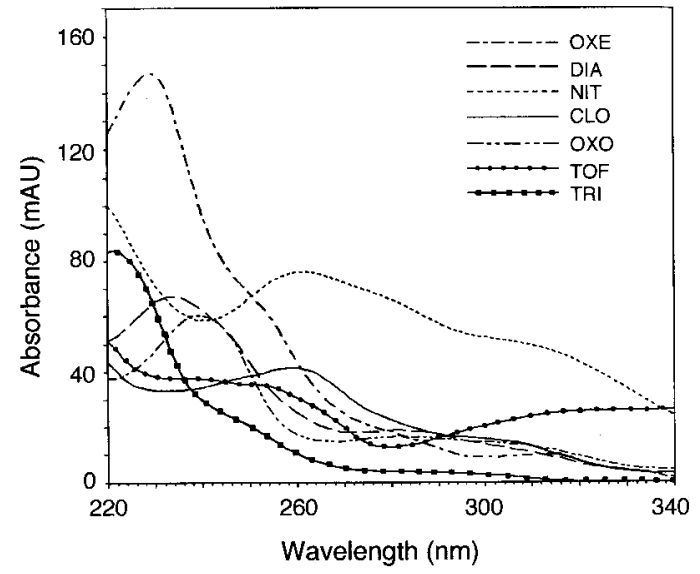

Fig. 2. UV Absorption Curves for Seven Benzodiazepines The concentration of each drug was $25 \mu \mathrm{g} / \mathrm{ml}$.

noise ratio of about 3 for standard solutions of BZDs, were 2.0 (OXO), 0.5 (NIT, OXE, TOF), 0.4 (TRI), 1.0 (CLO), and 0.5 (DIA), respectively. The relative standard deviations (RSD) after six identical repeated analyses at each concentration were $<0.8 \%$.

The accuracy and precision of the HPLC analysis of the seven BZDs were determined in recovery experiments. Commercial supplement placebos were spiked at concentration levels $(\mu \mathrm{g} / \mathrm{ml})$ of 19.4 (OXO), 5.2 (NIT), 5.1 (OXE), 11.6 (TOF), 1.4 (TRI), 9.7 (CLO), and 4.4 (DIA), respectively, and analyzed. Recoveries and standard deviations (S.D.) are presented in Table 1. The accuracy, calculated as the recovery of each BZD, was in the range of $98.0 \%$ to $104.8 \%$. The precision, calculated as RSD, was $<2.5 \%$. Figure 3 shows chromatograms obtained in the recovery experiment using a drug-free tablet-type 
Table 1. Accuracy and Precision of the Method for HPLC Analysis of Seven Benzodiazepines in Commercial Dietary Supplements $(n=5)$

\begin{tabular}{lcrrrrrr}
\hline \hline \multirow{2}{*}{ Type } & \multicolumn{1}{c}{ Drug } & \multicolumn{1}{c}{ OXE } & TOF & \multicolumn{1}{c}{ TRI } & CLO \\
\cline { 2 - 7 } & \multicolumn{1}{c}{ OXO } & \multicolumn{1}{c}{ NIT } & \multicolumn{1}{c}{ OXE } & DIA \\
\hline Tablet & $104.3 \pm 2.3$ & $102.8 \pm 1.9$ & $100.1 \pm 1.1$ & $102.1 \pm 1.2$ & $99.8 \pm 1.4$ & $100.1 \pm 0.9$ & $100.3 \pm 0.8$ \\
Capsule & $103.5 \pm 1.9$ & $102.2 \pm 1.8$ & $99.9 \pm 0.9$ & $100.9 \pm 1.0$ & $102.3 \pm 1.6$ & $101.2 \pm 0.7$ & $99.3 \pm 1.0$ \\
Teabag & $104.8 \pm 2.1$ & $103.6 \pm 1.1$ & $100.4 \pm 1.5$ & $102.3 \pm 1.4$ & $101.0 \pm 2.0$ & $98.5 \pm 0.6$ & $101.7 \pm 1.1$ \\
\hline
\end{tabular}

Each value represents recovery $(\%$, mean \pm S.D.).

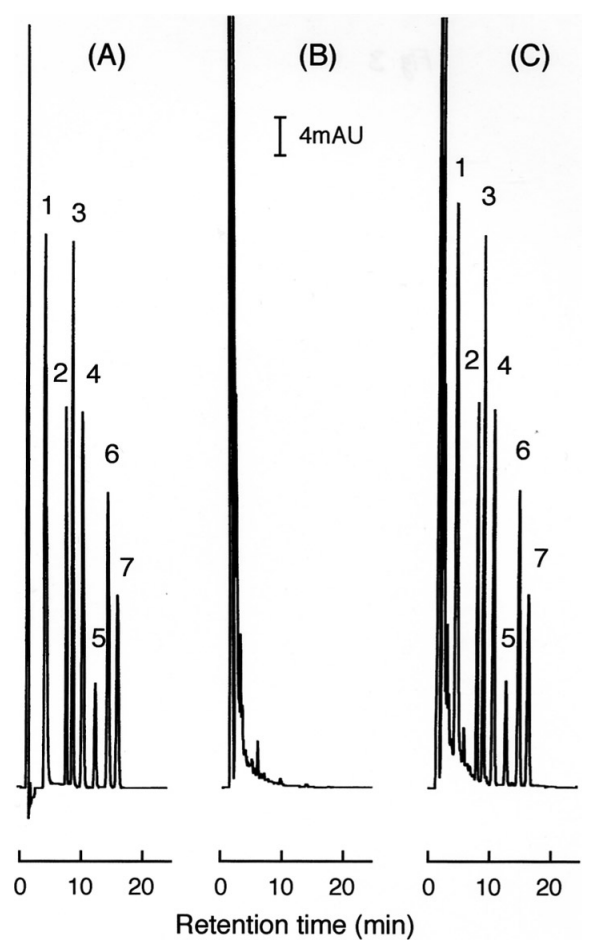

Fig. 3. HPLC Chromatograms of (A) Standard Solution, (B) Drug-Free Tablet, and (C) Tablet Spiked with Seven Benzodiazepines

Peaks: $1=$ OXO $(19.4 \mu \mathrm{g} / \mathrm{ml}), 2=$ NIT $(5.2 \mu \mathrm{g} / \mathrm{ml}), 3=$ OXE $(5.1 \mu \mathrm{g} / \mathrm{ml}), 4=$ TOF $(11.6 \mu \mathrm{g} / \mathrm{ml}), 5=$ TRI $(1.4 \mu \mathrm{g} / \mathrm{ml}), 6=$ CLO $(9.7 \mu \mathrm{g} / \mathrm{ml}), 7$ = DIA $(4.4 \mu \mathrm{g} / \mathrm{ml})$.

supplement. It is appropriate to analyze BZD-adulterated dietary supplements considering single oral doses of marketed preparations.

\section{Sample Analysis}

Fifteen samples were assayed using the procedure developed in this study. Seven BZDs were not detected in 14 samples using HPLC with the photodiode array. However, a peak in the capsule-type sample containing a yellow-white powder was identified as DIA in UV spectral analysis. The retention time of DIA in this HPLC analysis was $16.0 \mathrm{~min}$, and the peak in the capsule-type sample was identi-
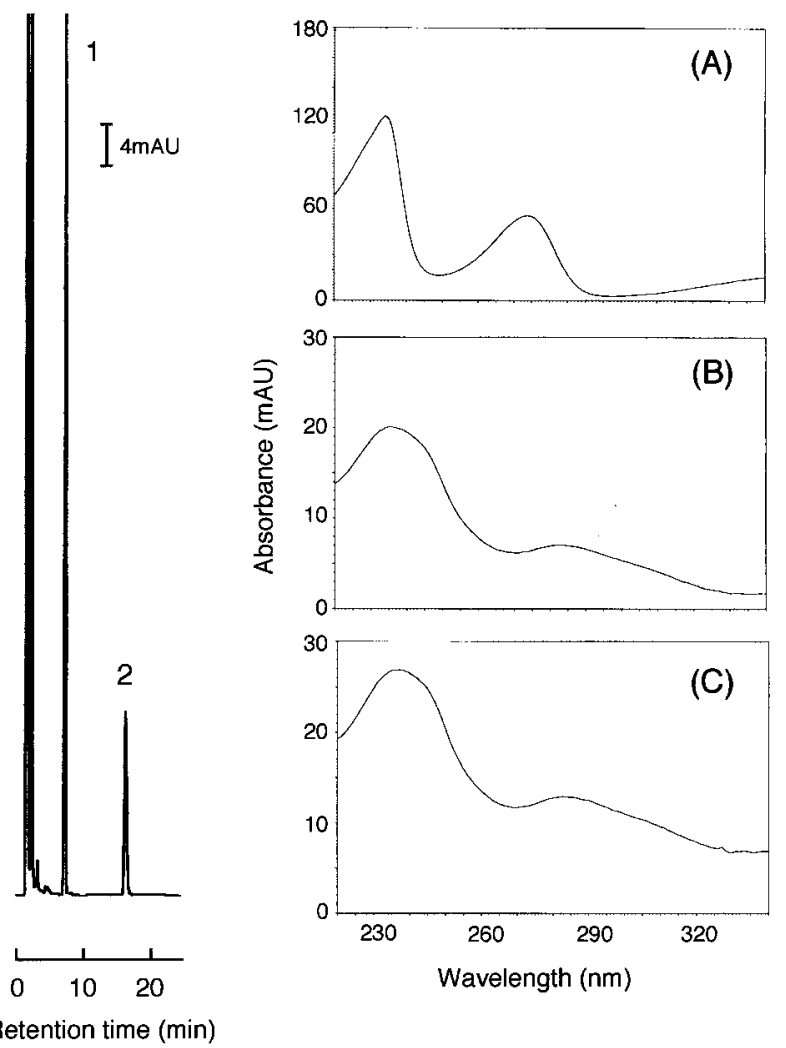

Fig. 4. HPLC Chromatogram of Capsule-Type Supplement, and UV Absorption Curves of (A) Peak = 1, (B) Peak = 2, and (C) DIA $(4.4 \mu \mathrm{g} / \mathrm{ml})$

fied. Figure 4 shows the UV spectra of the DIA standard and the capsule-type sample containing DIA monitored over the range of 220 to $340 \mathrm{~nm}$. The maximum wavelengths for DIA were 237 and $285 \mathrm{~nm}$. Quantitative analysis was performed at $225 \mathrm{~nm}$, and no interference was observed in the chromatograms. Figure 4 shows the HPLC chromatogram.

\section{Identification System for DIA in a Dietary Supple- ment}

TLC is widely used in pharmaceutical analytical laboratories for identity testing ${ }^{4,5,17)}$ because it is 


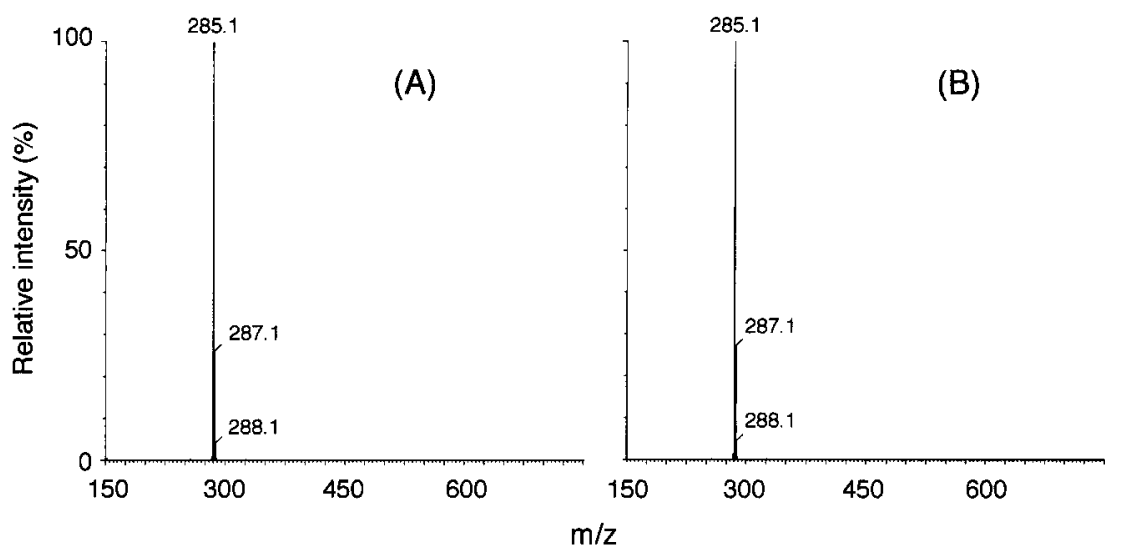

Fig. 5. Mass Spectra of (A) DIA Standard and (B) a Peak in Capsule-Type Supplement by ESI-LC/MS at Positive-Ion Mode

simple, rapid, robust, and inexpensive. The sample test solution was prepared by dilution with methanol and filtered to remove endogenous insoluble components. After development, a dark blue spot of DIA was observed under UV light at $254 \mathrm{~nm}$, and it was clearly resolved and well separated from the other components. The Rf value of DIA was 0.3 , and UV measurement allows reliable identification of DIA up to $0.1 \mu \mathrm{g}$.

LC/MS is a powerful qualitative technique for the accurate determination of molecular masses of analytes, because analyte identification on the basis of molecular mass is extremely selective. LC/MS using the ESI interface in positive-ion mode was used to determine the molecular marker ions of DIA. To improve sensitivity, the LC mobile phase was water/acetonitrile $(1: 1, \mathrm{v} / \mathrm{v})$. The retention time of DIA in this LC/MS analysis was $8.3 \mathrm{~min}$, and a peak in the supplement was identified. The protonated molecule $[\mathrm{M}+\mathrm{H}]+$ for DIA was found at $m / z 2285$. From a peak in the supplement, the protonated molecule $[\mathrm{M}+\mathrm{H}]+$ at $\mathrm{m} / \mathrm{z} 285$ was also observed. Figure 5 shows the mass spectra of the DIA standard and a supplement containing DIA generated in positiveion mode. The peak in the supplement was identified as DIA based on both mass and LC/MS spectra.

The sample was found to contain DIA $1.9 \mathrm{mg}$ per capsule using HPLC. A single oral dose of DIA is $2 \mathrm{mg}$ in Japan. ${ }^{18)}$ Such supplements are especially troublesome and frequently lead to overuse and have undesirable effects on the health of consumers.

In conclusion, an HPLC method for the simultaneous analysis of seven BZDs is presented for its application in the screening examination of dietary supplements as adulterants. After analysis of the 15 samples, DIA in the capsule-type supplement was detected. Finally, DIA was identified by a combination of three different analytical methods: HPLC/ photodiode array, TLC, and LC/MS. The identification of adulterants in dietary supplements poses an even greater challenge to laboratories required to conduct routine surveillance programs. Because of the great variation in the chemical properties, it is not possible to devise a single screening method to cover all medicinal ingredients. The procedure described here can provide an adequately broad analysis in a single run within $17 \mathrm{~min}$ and is available for the screening of seven BZDs in adulterated supplements with minimal sample preparation.

Acknowledgements This study was supported by a Health Sciences Research Grant from the Ministry of Health, Labor and Welfare, Japan.

\section{REFERENCES}

1) Thompson, R. D. and Carlson, M. (2000) Liquid chromatographic determination of dehydroepiandrosterone (DHEA) in dietary supplement products. $J$. AOAC Int., 43, 847-857.

2) Kojima, T., Kishi, M., Sekita, S. and Satake, M. (2001) Origin of sennosides in health teas including Malva leaves. J. Food Hyg. Soc. Jpn., 42, 202-205.

3) Kosaka, T. and Hamada, H. (2002) Analysis of thyroid hormones in health foods by LC/MS. J. Food Hyg. Soc. Jpn., 43, 225-229.

4) Mikami, E., Ohno, T. and Matsumoto, H. (2002) Simultaneous identification system for phentolamine and sildenafil as adulterants in soft drinks advertising roborant nutrition. Forensic Sci. Int., 130, 140146.

5) Kumasaka, K., Kojima, T., Doi, K. and Satoh, S. 
(2003) Analysis of the oral hypoglycemic agent, glibenclamide, in a health food. Yakugaku Zasshi, 123, 1049-1054.

6) Chang, Y.-S., Ku, Y.-R., Wen, K.-C. and Ho, L.-K. (2000) Analysis of synthetic gastrointestinal drugs in adulterated traditional chinese medicines by HPCE. J. Liq. Chromatogr. Relat. Technol., 23, 2009-2019.

7) Goto, T., Mikami, E., Ohno, T. and Matsumoto, H. (2002) Simultaneous analysis of four diuretic drugs by HPLC and its application to health food supplements advertising weight reduction. J. Food Hyg. Soc. Jpn., 43, 95-98.

8) Mikami, E., Ohno, T., Matsumoto, H. and Sekita, S. (2003) Detection of thyroxine in dietary supplements using an enzyme-linked immunosorbent assay. $J$. Health Sci., 49, 547-550.

9) Borrey, D., Meyer, E., Lambert, W., Van Peteghem, C. and De Leenheer, A. P. (2001) Simultaneous determination of fifteen low-dosed benzodiazepines in human urine by solid-phase extraction and gas chromatography-mass spectrometry. J. Chromatogr. B, 765, 187-197.

10) Guan, F., Seno, H., Ishii, A., Watanabe, K., Kumazawa, T., Hattori, H. and Suzuki, O. (1999) Solid-phase microextraction and GC-ECD of benzophenones for detection of benzodiazepines in urine. J. Anal. Toxicol., 23, 54-61.

11) Wilhelm, M., Battista, H. J. and Obendorf, D. (2000) Selective and sensitive assay for the determination of benzodiazepines by high-performance liquid chromatography with simultaneous ultraviolet and reductive electrochemical detection at the hanging mercury drop electrode. J. Chromatogr. A, 897,
215-225.

12) Gil-Agusti, M., Carda-Broch, S., Garcia-AlvarezCoque, M. C. and Esteve-Romero, J. (2000) Use of micellar mobile phases for the chromatographic determination of clorazepate, diazepam and diltiazem in pharmaceuticals. J. Chromatogr. Sci., 38, 521527.

13) Miki, A., Tatsuno, M., Katagi, M., Nishikawa, M. and Tsuchihashi, H. (2002) Simultaneous determination of eleven benzodiazepine hypnotics and eleven relevant metabolites in urine by columnswitching liquid chromatography-mass spectrometry. J. Anal. Toxicol., 26, 87-93.

14) The Japanese Pharmacopoeia, 14th Edition, Ministry of Health, Labour and Welfare, Tokyo, pp. 403404 (2001).

15) Mikami, E., Goto, T., Ohno, T., Matsumoto, H. and Nishida, M. (2000) Simultaneous analysis of naproxen, nabumetone and its major metabolite 6methoxy-2-naphthylacetic acid in pharmaceuticals and human urine by high-performance liquid chromatography. J. Pharm. Biomed. Anal., 23, 917-925.

16) Itoh, H., Nagano, T. and Takeyama, M. (2001) Effects of mosapride citrate on human plasma levels of motilin, gastrin, somatostatin and secretin. Biol. Pharm. Bull., 24, 1072-1075.

17) Ohno, T., Mikami, E. and Matsumoto, H. (2003) Identification of oil-soluble coal tar dyes in cosmetics using reversed-phase TLC/scanning densitometry. J. Health Sci., 49, 401-404.

18) Drugs in Japan, Ethical Drugs, 27th ed. (Japan Pharmaceutical Information Center, Ed.), Jiho Inc., Tokyo, pp. 887-890 (2003). 